\title{
Generation of 3.5 W of diffraction-limited green light from SHG of a single tapered diode laser in a cascade of nonlinear crystals
}

Hansen, Anders Kragh; Jensen, Ole Bjarlin; Sumpf, Bernd; Erbert, Götz; Unterhuber, Angelika; Drexler, Wolfgang; Andersen, Peter E.; Petersen, Paul Michael

\section{Published in:}

Proceedings of SPIE

Link to article, DOI:

$10.1117 / 12.2038965$

Publication date:

2014

Document Version

Peer reviewed version

Link back to DTU Orbit

Citation (APA):

Hansen, A. K., Jensen, O. B., Sumpf, B., Erbert, G., Unterhuber, A., Drexler, W., Andersen, P. E., \& Petersen, P. M. (2014). Generation of $3.5 \mathrm{~W}$ of diffraction-limited green light from SHG of a single tapered diode laser in a cascade of nonlinear crystals. In Proceedings of SPIE (Vol. 8964). SPIE - International Society for Optical Engineering. Proceedings of SPIE - The International Society for Optical Engineering https://doi.org/10.1117/12.2038965

\section{General rights}

Copyright and moral rights for the publications made accessible in the public portal are retained by the authors and/or other copyright owners and it is a condition of accessing publications that users recognise and abide by the legal requirements associated with these rights.

- Users may download and print one copy of any publication from the public portal for the purpose of private study or research.

- You may not further distribute the material or use it for any profit-making activity or commercial gain

- You may freely distribute the URL identifying the publication in the public portal 


\title{
Generation of 3.5 W of diffraction-limited green light from SHG of a single tapered diode laser in a cascade of nonlinear crystals
}

\author{
Anders K. Hansen*a, Ole B. Jensen ${ }^{\mathrm{a}}$, Bernd Sumpf ${ }^{\mathrm{b}}$, Götz Erbert ${ }^{\mathrm{b}}$, Angelika Unterhuber ${ }^{\mathrm{c}}$, Wolfgang \\ Drexler $^{\mathrm{c}}$, Peter E. Andersen ${ }^{\mathrm{a}}$, Paul Michael Petersen ${ }^{\mathrm{a}}$ \\ ${ }^{a}$ Dept. of Photonics Engineering, Technical University of Denmark, 399 Frederiksborgvej, Roskilde,

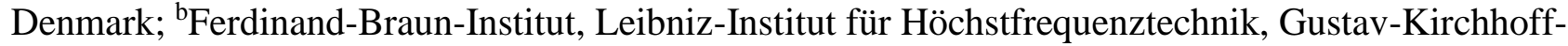 \\ Straße 4, 12489 Berlin, Germany; ${ }^{\circ}$ Medical University of Vienna, Center for Medical Physics and \\ Biomedical Engineering, Waehringer Guertel 18-20, 1090 Vienna, Austria
}

*ankrh@fotonik.dtu.dk; phone +45 46774586

\begin{abstract}
Many applications, e.g., within biomedicine stand to benefit greatly from the development of diode laser-based multiWatt efficient compact green laser sources. The low power of existing diode lasers in the green area (about $100 \mathrm{~mW})$ means that the most promising approach remains nonlinear frequency conversion of infrared tapered diode lasers.

Here, we describe the generation of $3.5 \mathrm{~W}$ of diffraction-limited green light from SHG of a single tapered diode laser, itself yielding $10 \mathrm{~W}$ at $1063 \mathrm{~nm}$. This SHG is performed in single pass through a cascade of two PPMgO:LN crystals with re-focusing and dispersion compensating optics between the two nonlinear crystals. In the low-power limit, such a cascade of two crystals has the theoretical potential for generation of four times as much power as a single crystal without adding significantly to the complexity of the system. The experimentally achieved power of $3.5 \mathrm{~W}$ corresponds to a power enhancement greater than 2 compared to SHG in each of the crystals individually and is the highest visible output power generated by frequency conversion of a single diode laser.
\end{abstract}

Such laser sources provide the necessary pump power for biophotonics applications, such as optical coherence tomography or multimodal imaging devices, e.g., FTCARS-OCT, based on a strongly pumped ultrafast Ti:Sapphire laser.

Keywords: Nonlinear Optics, Second Harmonic Generation, Diffraction-Limited Light, Semiconductor Lasers, Tapered Diode Lasers, Green Lasers

\section{INTRODUCTION}

High-brightness infrared tapered diode lasers ${ }^{1}$ have recently been efficiently single-pass frequency doubled to the green spectral region $^{2}$ and have been demonstrated as good high-power, diffraction limited green laser sources. Such laser sources are very important for a variety of applications, including pumping of Ti:sapphire lasers ${ }^{3,4}$ and for direct use in dermatology. Basing such sources on diode lasers rather than solid state lasers is especially advantageous because of the high electro-optical efficiencies of diode lasers as well as the possibility of wavelength tunability and selectability through the material composition of the gain medium of diode lasers. Since frequency doubled diode lasers can additionally be implemented very compactly and cost-efficiently, they are particularly attractive for integration in more complex laser systems such as Ti:sapphire systems for biomedical imaging.

Such systems could make use of short femtosecond pulses for, e.g., two-photon microscopy ${ }^{5,6}$, coherent anti-Stokes Raman scattering $^{7}$ (CARS) or high resolution optical coherence tomography ${ }^{8}$ (OCT). The prospect of multimodal biomedicinal imaging is one of particular interest, with promise of providing better and more reliable diagnoses. Multimodal imaging does, however, place increased demands on the power of the Ti:sapphire laser in the system and thus, in turn, the power of the green pump laser. 
The quest for higher green power output from frequency doubling of diode lasers is still ongoing ${ }^{9,10}$, and a crucial parameter to obtain higher power is the nonlinear conversion efficiency. While high nonlinear conversion efficiencies can be obtained using intracavity or external cavity setups, the added complexity of establishing and maintaining the precisely aligned cavities makes this approach less appealing than a single-pass configuration in which infrared laser light is generated monolithically in a laser diode and sent single-pass through a nonlinear crystal with high nonlinearity such as periodically poled lithium niobate (PPLN) to generate green light.

Until now, the highest power achieved in such a single-pass configuration using a single tapered diode laser ${ }^{2}$ has been $1.5 \mathrm{~W}$ of green radiation at $532 \mathrm{~nm}$, with the tapered diode laser emitting up to $12 \mathrm{~W}$ of infrared light at $1063 \mathrm{~nm}$ and using PPLN as the nonlinear crystal. In another setup, two such infrared diode lasers were spectrally combined in a volume Bragg grating and sum frequency generation was performed in PPLN, yielding 3.9 W of diffraction-limited green light ${ }^{11}$.

One method of increasing the conversion efficiency of SHG compared to a single pass setup is to send the beam through the nonlinear crystal more than once ${ }^{12}$, while still not establishing a cavity. Such multi-pass setups require very careful alignment of the beam to avoid feedback to the sensitive diode laser and can be challenging in periodically poled crystals due to the crystals' small size.

Another method involves the use of waveguides of nonlinear media ${ }^{13}$. The confinement of the fundamental and SHG beams provided by the waveguide increases the efficiency of the process. The drawback is the difficulty in coupling into the waveguide and low damage thresholds for the system, currently restricting output powers.

Similar to these other two methods is that of using several nonlinear crystals in a sequence, known as a cascade, in which the beam passes through each crystal only once. In a cascade, the beam is focused into the first crystal and re-focusing optics create a new focus in each of the subsequent crystals. Cascade SHG was first demonstrated in 1997 by D. Fluck and P. Günter ${ }^{14}$, using lenses as the re-focusing optics and using a sequence of up to three nonlinear crystals. They referred to the technique as "lens waveguiding in a cascade of crystals". This technique inherits the favorable scaling laws of confined beams while avoiding the limitations caused by low damage thresholds of waveguides. At the same time, it avoids the physical space and/or optical feedback issues of multi-pass configurations as well as allowing independent phase matching control of each crystal. Cascaded frequency conversion is starting to become more widely adopted, with many groups utilizing the setup in recent years ${ }^{15-21}$.

\section{THEORY}

Fluck and Günter showed theoretically how the output power in the limit of low depletion of the fundamental beam scales with the square of the number of crystals. In the case of a cascade of $N$ identical crystals of length $L$ with optimal focusing in each individual crystal, the output power in the low depletion limit is

$$
P_{2}=\bar{\eta} L N^{2} P_{1}^{2}
$$

where $P_{2}$ is the total output second harmonic power, $\bar{\eta}$ is the normalized conversion efficiency for a single pass and $P_{1}$ is the fundamental power incident on the system. From this it is evident that adding a second crystal of the same type to an existing single-crystal setup can yield an enhancement of the output power of up to a factor of four.

Recall that for collimated beams the scaling in crystal length is quadratic, as is also the case in waveguides because of the confinement of the light. When focusing into a crystal, diffraction reduces the scaling factor to linear as described in the theory of Boyd and Kleinman ${ }^{22}$. Re-focusing into subsequent crystals is a way around that scaling law by introducing the $N^{2}$ scaling factor.

As with single-crystal SHG, fundamental depletion can be taken into account using

$$
P_{2}=P_{1} \tanh ^{2} \sqrt{\eta P_{1}}
$$


where, in the case of a cascade, $\eta=\bar{\eta} L N^{2} . \eta$ is the system's nonlinear conversion efficiency in the low-depletion limit.

While Fluck and Günter as well as some later authors used lenses as the re-focusing optics, others had chosen spherical mirrors to avoid chromatic aberrations, allowing the SHG and fundamental beams to co-propagate better and re-focus in the same plane in each subsequent crystal, aiding the SHG process.

Similarly to the well-known phase matching condition that must still be satisfied within each crystal, it is necessary to compensate for any dispersion between the fundamental and SHG beams that arises between each pair of crystals. This arises due to the specifics of the poling at the ends of the crystals, dispersion in air and dispersion in the re-focusing optics used. The original setup of Fluck and Günter as well as some of the setups of the most recent applications used a transparent plane plate, rotatable around either the vertical or lateral axis, placed between each pair of crystals to adjust the dispersion compensation. Others have used the dispersion of air in a setup where the path length of travel through air was adjustable with a translation stage.

For a $3 \mathrm{~mm}$ thick plate of BK-7 glass with parallel facets, the dispersion compensation as a function of the angle of tilt of the transparent plate can be found by using Snell's law of refraction and calculating the difference in optical path length of the fundamental and SHG beams. The SHG power after the second crystal is then a result of the interference of the SHG E-field generated in the first crystal and the SHG E-field generated in the second crystal. The simulated output power in the low-depletion regime as a function of plate angle is shown in fig. 1. The difference in the lateral displacement of the fundamental and SHG beams upon passage through the plate is on the order of micrometers and its effect has been neglected, since it occurs at a point where the beam is collimated with a diameter of more than a millimeter. Likewise, reflection loss at the interfaces has been omitted.

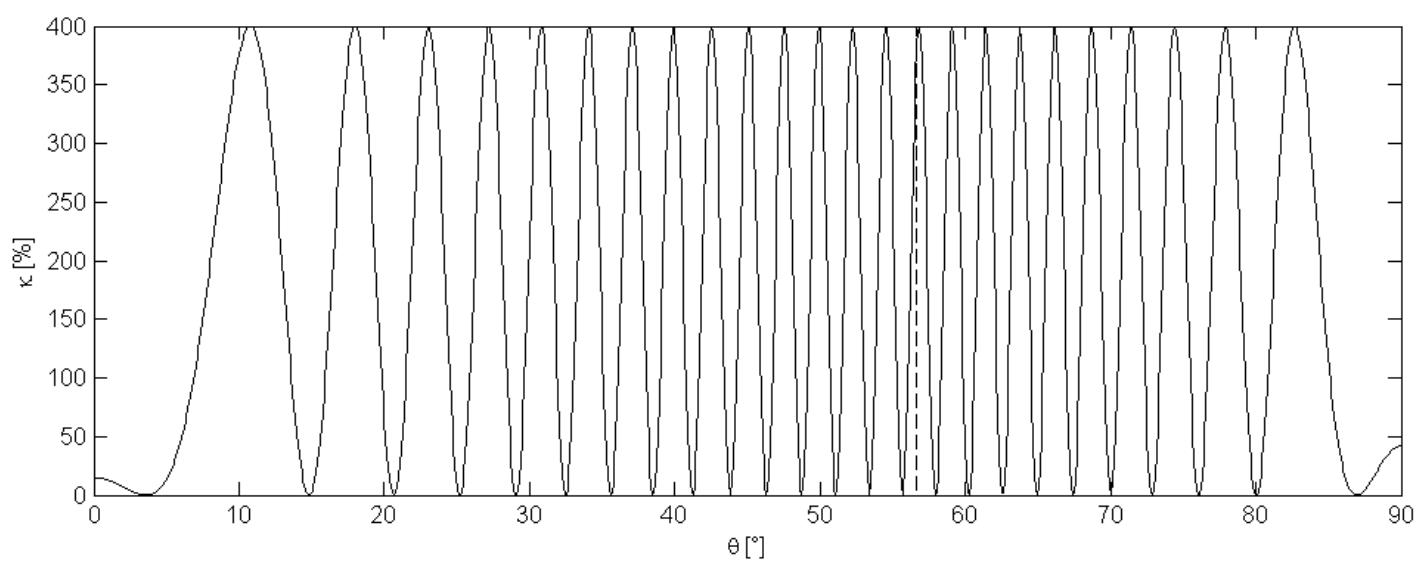

Figure 1. Simulation of the cascade enhancement factor $\kappa$ defined as the ratio of $\eta$ of the whole cascade to $\eta$ of only the first crystal. The shown example is for a Gaussian beam at $1063 \mathrm{~nm}$ sent through a cascade of two identical crystals, with reflections at the dispersive plate interfaces as well as a small displacement between the two beams neglected. The dashed line shows Brewster's angle and the plate is taken to be BK-7 glass. Imperfections in the crystals, slightly different focusing, astigmatism and absorption-induced heating effects will cause the real curve to deviate from the theoretical curve by exhibiting a maximum lower than $400 \%$ and a minimum higher than $0 \%$.

As an alternative to the use of a transparent plate, one can use the dispersion of air to adjust the dispersion between the crystals. The path length required for one period of retardation is $13.1 \mathrm{~cm}$ for air at room temperature and atmospheric pressure $^{23}$. If one utilizes a setup with a delay line for adjustment of this dispersion, one must align the delay line carefully to avoid shifting the alignment of the beam when changing the delay path length. The collimation of the beam in the delay line must also be carefully optimized. 


\section{EXPERIMENT}

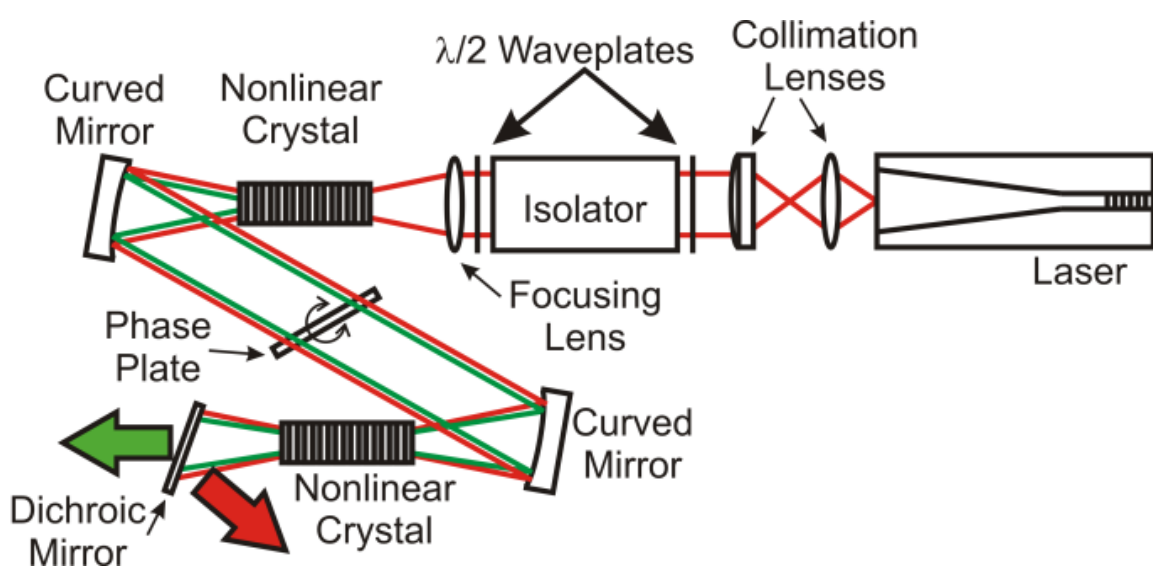

Figure 2. The experimental setup is shown here in sketch form. The infrared-emitting diode laser is collimated with a pair of anti-reflection coated lenses, sent through an optical isolator with a half-wave plate before and after for polarization rotation, and focused into the first crystal. A pair of curved mirrors re-focus the beam at low angles of incidence into the second crystal, with a transparent phase plate placed between the curved mirrors for dispersion compensation. A dichroic mirror filters away the infrared light after the second crystal, allowing the SHG light to be measured with a power meter after the dichroic mirror.

The experimental setup is shown in fig. 2. The tapered diode laser emitted infrared light at a wavelength of $1063 \mathrm{~nm}$. The emission was collimated in the fast axis after passage through an aspheric lens of focal length $3.1 \mathrm{~mm}$ and in the slow axis after further passage through a cylinder lens of focal length $15 \mathrm{~mm}$. The $1 / \mathrm{e}^{2}$ beam diameter of the collimated beam was $1.1 \mathrm{~mm}$ along both axes.

The injection currents used for the ridge waveguide section and tapered amplifier section were $300 \mathrm{~mA}$ and $16 \mathrm{~A}$, respectively. The laser diode was temperature stabilized with a Peltier element to a temperature of 20.00 degrees centigrade. At these operation parameters, the infrared power after collimation was $10.5 \mathrm{~W}$.

The light was then passed though an optical isolator protecting the diode from optical feedback, with half-wave plates rotating the polarization of the beam before and after the optical isolator. The infrared power after the optical isolator and wave plates was $10.0 \mathrm{~W}$.

Two PPLN crystals were used for the cascade, each $30 \mathrm{~mm}$ long and mounted in closed-top temperature adjustable aluminum ovens. The entrance and exit facets were cut for a 0 degree angle of incidence. Focusing into the first crystal was performed with an achromatic lens of focal length $60 \mathrm{~mm}$, which had been experimentally found to be the optimal focal length for highest SHG power generated in a single-crystal setup. For characterization, each of the two crystals was individually tested in this focus. Crystal \#1 yielded an output of $1.78 \mathrm{~W}$ of green light at an infrared input power of 10.0 $\mathrm{W}$, corresponding to a non-depleted efficiency of $\eta=2.0 \% / \mathrm{W}$ using equation (2). Tested in the same way in the place of crystal \#1, crystal \#2 showed a green output of $1.80 \mathrm{~W}$ at an infrared input of $10.0 \mathrm{~W}$, also corresponding to a nondepleted efficiency $\eta=2.0 \% / \mathrm{W}$.

With crystal \#1 once again placed in the focus, the diverging beam exiting the crystal, consisting of both infrared and SHG light, was collimated with a spherically concave mirror with radius of curvature $\mathrm{R}=300 \mathrm{~mm}$ (focal length $\mathrm{f}=150$ $\mathrm{mm}$ ) at an angle of incidence of 10 degrees. The angle of incidence was chosen as low as possible within the physical constraints of the setup in order to minimize astigmatism and other imaging aberrations caused by hitting the mirror offaxis. The mirror was coated for high reflectivity at $1064 \mathrm{~nm}$ and at $532 \mathrm{~nm}$.

A $3 \mathrm{~mm}$ thick transparent plate of uncoated BK-7 glass was inserted into the collimated beam and rotated around the lateral axis to bring the plate to Brewster's angle of incidence to minimize reflection loss for the vertically polarized fundamental and SHG light. Additional tuning of a few degrees around Brewster's angle was possible to adjust the dispersion compensation.

After passage through the transparent plate, the beam was re-focused into crystal \#2 using a concave mirror identical to the first one, with radius of curvature $\mathrm{R}=300 \mathrm{~mm}$ and the same angle of incidence. 
After individual optimization of the phase matching temperatures of the two crystals and optimization of the angle of the dispersive plate, the optimal SHG power after crystal \#2 was measured to be $3.48 \mathrm{~W}$, corresponding to a conversion efficiency in the non-depletion regime of $\eta=4.6 \% / \mathrm{W}$. The enhancement relative to the single-crystal case is a factor of 2.3, greater than the sum of SHG achievable from only the first crystal and SHG achievable from only the second crystal.

The SHG output power as a function of the rotation angle of the dispersive plate was measured and is shown in fig. 3.

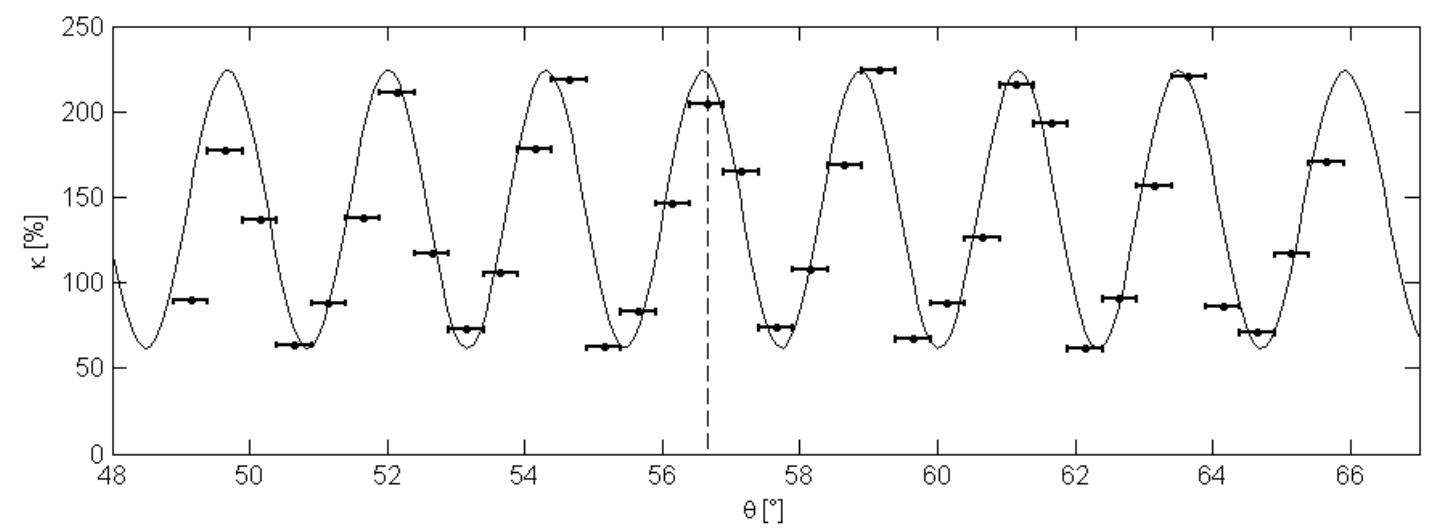

Figure 3. Enhancement factor $\kappa$, defined as in fig. 1, as a function of dispersive plate angle of rotation around the lateral axis. The solid curve is the theoretical curve of fig. 1 shifted and scaled to the same minimum and maximum values as the data points. Brewster's angle is shown with the dashed line. Good agreement is observed between the theoretical period close to Brewster's angle of $2.3^{\circ}$ and the experiment.

From fig. 3 we see agreement between the calculated and measured periodicity of the dispersion compensation of $2.3^{\circ}$ close to Brewster's angle. The lowest power achievable when tuning the dispersive plate is $1.14 \mathrm{~W}$, which is $33 \%$ of the maximum value. This lowest output is lower than the SHG power directly after the first crystal, showing the effect of the destructive interference between SHG generated in the two crystals.

\section{CONCLUSIONS AND OUTLOOK}

Generating $3.5 \mathrm{~W}$ of diffraction-limited radiation at $532 \mathrm{~nm}$ from a single tapered diode laser is an important step in the ongoing quest for higher powers in the green spectral region. The use of nonlinear crystals in a cascade allows an increase of the nonlinear conversion efficiency beyond that achievable with single crystals, with a highly advantageous scaling law. In this work, an enhancement of a factor of 2.3 was observed compared to the single-crystal case, with potential for further optimization to get closer to the theoretical ideal case in which the enhancement is a factor of 4 . Future implementations of the cascade may include crystals longer than $30 \mathrm{~mm}$, a third crystal and/or tapered diode lasers with higher power or better beam quality.

The laser system described in this work has great potential as a pump source for a Ti:sapphire laser in a multimodal system such as FTCARS-OCT. The increased power for pumping the mode-locked Ti:sapphire laser, will be crucially important for increasing bandwidth, increasing resolution, decreasing acquisition time, and accessing the unique benefits of a multimodal system.

Compared to solid state lasers, diode lasers lend themselves particularly well to mass production and miniaturization, and the powers and beam qualities of infrared tapered diode lasers are expected to see continuing improvements for the foreseeable future.

\section{REFERENCES}

[1] Sumpf, B., Hasler, K.-H., Adamiec, P., Bugge, F., Dittmar, F., Fricke, J., Wenzel, H., Zorn, M., Erbert, G. and Trankle, G., "High-Brightness Quantum Well Tapered Lasers," IEEE J. Sel. Top. Quantum Electron. 15(3), 1009-1020 (2009). 
[2] Jensen, O. B., Andersen, P. E., Sumpf, B., Hasler, K.-H., Erbert, G. and Petersen, P. M., "1.5 W green light generation by single-pass second harmonic generation of a single-frequency tapered diode laser," Opt. Express 17, 6532-6539 (2009).

[3] Unterhuber, A., Povazay, B., Müller, A., Jensen, O. B., Duelk, M., Le, T., Petersen, P. M., Velez, C., Esmaeelpour, M., Andersen, P. E. and W. Drexler, "Simultaneous dual wavelength eye-tracked ultrahigh resolution retinal and choroidal optical coherence tomography," Optics Letters 38, 4312-4315 (2013).

[4] Müller, A., Jensen, O. B., Unterhuber, A., Le, T., Stingl, A., Hasler, K.-H., Sumpf, B., Erbert, G., Andersen, P. E. and Petersen, P. M., "Frequency-doubled DBR-tapered diode laser for direct pumping of Ti:sapphire lasers generating sub-20 fs pulses," Opt. Express 19, 12156-12163 (2011).

[5] König, K., "Clinical multiphoton tomography," J. Biophoton. 1, 13-23 (2008).

[6] Denk, W., Strickler, J. H. and Webb, W. W., "Two-photon laser scanning fluorescence microscopy," Science 248, 73-76 (1990).

[7] Maker, P. D. and Terhune, R. W., "Study of Optical Effects Due to an Induced Polarization Third Order in the Electric Field Strength,” Phys. Rev. 137, A801-A818 (1965).

[8] Huang, D., Swanson, E.A., Lin, C.P., Schuman, J.S., Stinson, W.G., Chang, W., Hee, M.R., Flotte, T., Gregory, K., Puliafito, C.A., Fujimoto, J.G., “Optical coherence tomography,” Science 254, 1178-1181 (1991).

[9] Jensen, O. B., Hansen, A. K., Müller, A., Sumpf, B., Unterhuber, A., Drexler, W., Petersen, P. M. and Andersen, P. E., "Power Scaling of Nonlinear Frequency Converted Tapered Diode Lasers for Biophotonics," IEEE J. Sel. Top. Quantum Electron. 20, 7100515 (2014).

[10] Fiebig, C., Fricke, J., Uebernickel, M., Jedrzejczyk, D., Sahm, A. and Paschke, K., "Watt-Class Green-Emitting Laser Modules Using Direct Second Harmonic Generation of Diode Laser Radiation," Opt. Rev. 19, 405-408 (2012).

[11] Müller, A., Jensen, O. B., Hasler, K.-H., Sumpf, B., Erbert, G., Andersen, P. E. and Petersen, P. M., "Efficient concept for generation of diffraction-limited green light by sum-frequency generation of spectrally combined tapered diode lasers," Opt. Lett. 37, 3753-3755 (2012).

[12] Spiekermann, S., Laurell, F., Pasiskevicius, V., Karlsson, H. and Freitag, I., “Optimizing non-resonant frequency conversion in periodically poled media,” Appl. Phys. B, 79(2), 211-219 (2004).

[13] Bhatia, V., Bauco, A. S., Oubei, H. M. and Loeber, D. A. S., "Efficient green lasers for high-resolution scanning micro-projector displays," Proc. SPIE, P. E. Powers 7582, 758205-758205-12 (2010).

[14]Fluck, D. and Günter, P., "Efficient second-harmonic generation by lens wave-guiding in $\mathrm{KNbO}_{3}$ crystals," Optics Communications 147, 305-308 (1998).

[15] Samanta, G. K., Kumar, S. C., Devi, K. and Ebrahim-Zadeh, M., "Multicrystal, continuous-wave, single-pass second-harmonic generation with 56\% efficiency," Opt. Lett. 35, 3513-3515 (2010).

[16] Kumar, S. C., Samanta, G. K., Devi, K. and Ebrahim-Zadeh, M., "High-efficiency, multicrystal, single-pass, continuous-wave second harmonic generation," Opt. Express 19, 11152-11169 (2011).

[17] Chiow, S.-w., Kovachy, T., Hogan, J. M. and Kasevich, M. A., "Generation of 43 W of quasi-continuous 780 $\mathrm{nm}$ laser light via high-efficiency, single-pass frequency doubling in periodically poled lithium niobate crystals," Opt. Lett. 37, 3861-3863 (2012).

[18] Vasilyev, S., Nevsky, A., Ernsting, I., Hansen, M., Shen, J. and Schiller, S., "Compact all-solid-state continuous-wave single-frequency UV source with frequency stabilization for laser cooling of $\mathrm{Be}^{+}$ions," Applied Physics B 103, 27-33 (2011).

[19] Garai, S. K. and Mukhopadhyay, S., "A method of optical implementation of frequency encoded different logic operations using second harmonic and difference frequency generation techniques in non-linear material," Optik - International Journal for Light and Electron Optics 121, 715-721 (2010).

[20]Lienhart, F., Boussen, S., Carraz, O., Zahzam, N., Bidel, Y. and Bresson, A., "Compact and robust laser system for rubidium laser cooling based on the frequency doubling of a fiber bench at $1560 \mathrm{~nm}$," Applied Physics B 89, 177-180 (2007).

[21] Thompson, R., Tu, M., Aveline, D., Lundblad, N. and Maleki, L., "High power single frequency 780nm laser source generated from frequency doubling of a seeded fiber amplifier in a cascade of PPLN crystals," Opt. Express 11, 1709-1713 (2003).

[22] Boyd, G. and Kleinman, D., "Parametric Interaction of Focused Gaussian Light Beams," Journal of Applied Physics 39, 3597-3639 (1968).

[23] Yarborough, J. M., Falk, J. and Hitz, C. B., "Enhancement of Optical Second Harmonic Generation By Utilizing the Dispersion of Air,” Appl. Phys. Lett. 18(3), 70-73 (1971). 